Rev Colomb Cir. 2018;33:345-52

https://doi.org/10.30944/20117582.81

\title{
Proyecto Urkunina 5000 \\ Investigación de la prevalencia de lesiones precursoras y del efecto de la erradicación de Helicobacter pylori como prevención primaria del cáncer gástrico en el departamento de Nariño
}

\author{
URKUNINA Project 5000 \\ Investigation on the prevalence of precursor lesions and of the effect of \\ eradication of Helicobacter pylorl as primary prevention of gastric cancer \\ in the state of Nariño, Colombia \\ Álvaro Bedoya-Urresta1, Yomaira Yépez², Diana Calvache², Yenny Cifuentes², Nubia Lucero², \\ Patricia González², Álvaro Bedoya G. ${ }^{2}$, Eliana Manosalva², Teresa Martínez ${ }^{3}$, Arecio Peñalosa ${ }^{4}$, \\ Blanca Piazuelo ${ }^{5}$ \\ 1 Director Científico, Centro de Investigación de Enfermedades digestivas y nutricionales, CIEDYN, Hospital Universitario \\ Departamental de Nariño, Pasto, Colombia \\ 2 Coinvestigador, Centro de Investigación de Enfermedades digestivas y nutricionales, CIEDYN, Hospital Universitario Departamental \\ de Nariño, Pasto, Colombia \\ 3 Instituto Nacional de Cancerología, Bogotá, D.C., Colombia \\ 4 Jefe, Sección de Gastroenterología y Endoscopia, Fundación Universitaria Ciencias de la Salud, FUCS, Bogotá, D.C., Colombia \\ 5 Médica patóloga, investigadora, Vanderbilt University, Nashville, TN; United States \\ Resumen del Proyecto URKUNINA 5000 aprobado por COLCIENCIAS \\ Entidades cooperantes: \\ - Centro de Investigación de Enfermedades Digestivas y Nutricionales, Hospital Universitario Departamental de Nariño \\ - Gobernación de Nariño \\ - Instituto Nacional de Cancerología \\ - Asociación Colombiana de Cirugía \\ - Fundación Universitaria de Ciencias de la Salud
}

\section{Introducción}

El proyecto Urkunina 5000 fue aprobado por el Órgano Colegiado de Administración y Decisión del Fondo de Ciencia, Tecnología e Innovación del Sistema General de Regalías,
Colciencias, mediante el Acuerdo No. 57 del 9 de junio de 2017 ,

'Urkunina' es un vocablo indígena que significa 'montaña de fuego', en honor al volcán Galeras, centinela de San Juan de Pasto (Nariño,

Palabras clave: Helicobacter pylori; neoplasias gástricas; investigación; detección precóz del cáncer; prevención primaria.

Key words: Helicobacter pylori; stomach neoplasms; research; early detection of cancer; primary prevention.

Fecha de recibido: 21/06/201/ - Fecha aceptación: 23/07/2018

Correspondencia: Álvaro Bedoya-Urresta, MD, Clínica San Juan de Pasto, Cll. 16 \#29 - 63, San Juan de Pasto

Correo electrónico: alvarobedoya2@yahoo.es

Citar: Bedoya Urresta A, Yépez Y, Calvache D, Cifuentes Y, Lucero N, González P, et al. Proyecto Urkunina 5000 - Investigación de la prevalencia de lesiones precursoras y del efecto de la erradicación de Helicobacter pylori como prevención primaria del cáncer gástrico en el departamento de Nariño. Rev Colomb Cir. 2018;33:345-52. https://doi.org/10.30944/20117582.81

Este es un artículo de acceso abierto bajo una Licencia Creative Commons - BY-NC-ND https://creativecommons.org/licenses/by-nc-nd/4.0/deed.es 
Colombia), ciudad sede del proyecto; y 5000, hace relación a los participantes voluntarios de los 55 municipios del área andina nariñense, zona de alto riesgo de cáncer gástrico.

El departamento de Nariño está localizado al suroccidente de Colombia, frontera con la república de Ecuador y salida al océano Pacífico, con I'800.00o habitantes, aproximadamente, y 64 municipios, de los cuales 55 están localizados en la zona andina, que representa el 8I,7 \% de la población nariñense. El departamento presenta una situación geográfica, social y étnica especial: cuenta con una zona andina montañosa alta que presenta una elevada incidencia de cáncer gástrico y una zona costera baja que, de manera contraria, presenta una incidencia baja de cáncer gástrico. Este pequeño territorio de $32.265 \mathrm{~km}^{2}$ presenta tres zonas definidas de cáncer gástrico, lo que lo hace especial y tal vez único en el mundo:

- Dos zonas conocidas como rojas, al norte y el suroccidente, con una de las incidencias más altas del mundo (I50 por IOo.00o habitantes), con alta prevalencia de lesiones precursoras de malignidad e infección por Helicobacter pylori;
- una zona amarilla, que corresponde a su capital (San Juan de Pasto), y municipios circunvecinos, con una incidencia de 46 por 100.000 habitantes,

- y una zona verde en la costa del Pacífico, con una incidencia de 6 por Ioo.0oo habitantes, una menor prevalencia de lesiones precursoras de malignidad y alta prevalencia de infección por H. pylori, conocida actualmente como el enigma nariñense o colombiano ${ }^{\mathrm{I}-3}$ (figura 1 ).

\section{Justificación}

Lo anterior se vio reflejado en un estudio publicado en el año 2008 por W. Sánchez ${ }^{4}$, y que se ha mantenido hasta la actualidad, confirmando que la epidemiología del cáncer gástrico no ha cambiado significativamente en nuestro país. En este estudio, se afirma que el cáncer gástrico es de predominio andino y las áreas con mayor registro de mortalidad corresponden a los departamentos de Nariño y Cauca (figura 2). Lo anterior se refuerza con lo reportado en la literatura médica con respecto a la supervivencia a largo plazo (5 años), que en Nariño y Colombia, con respecto a otros países del mundo, sigue siendo muy baja (figura 3).

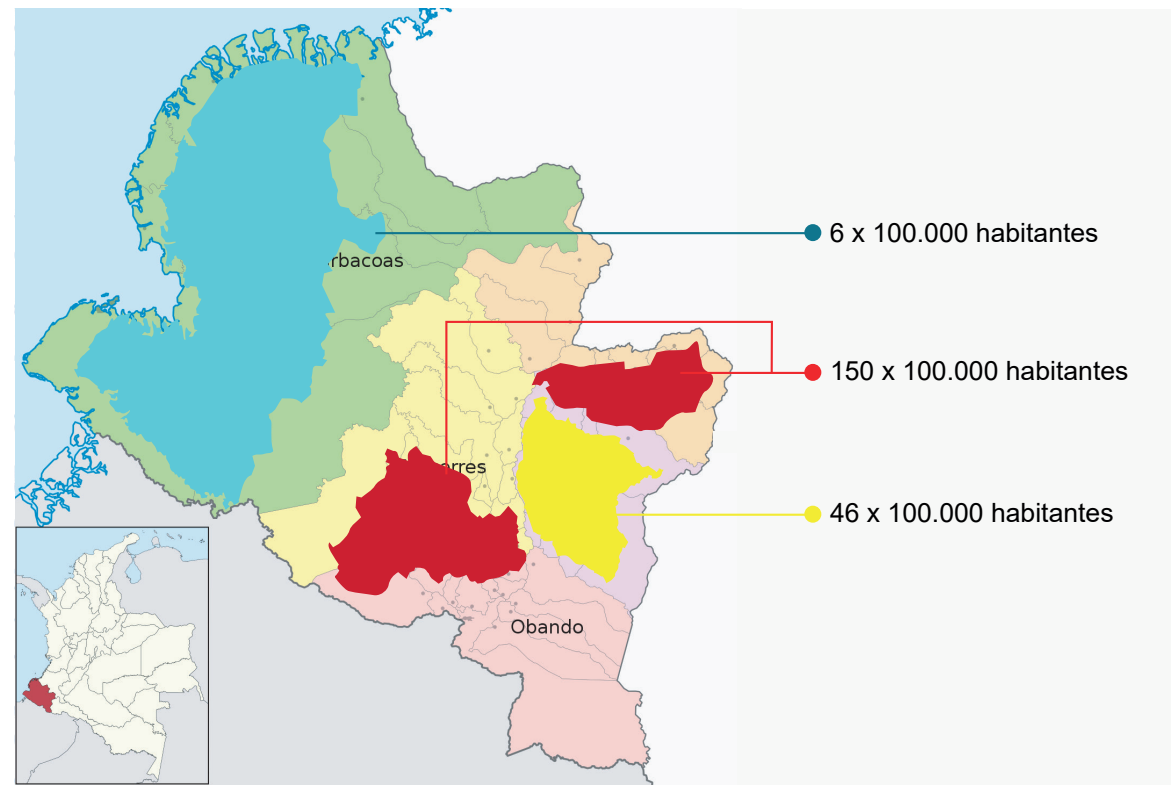

Figura 1. Áreas de cáncer gástrico en Nariño 


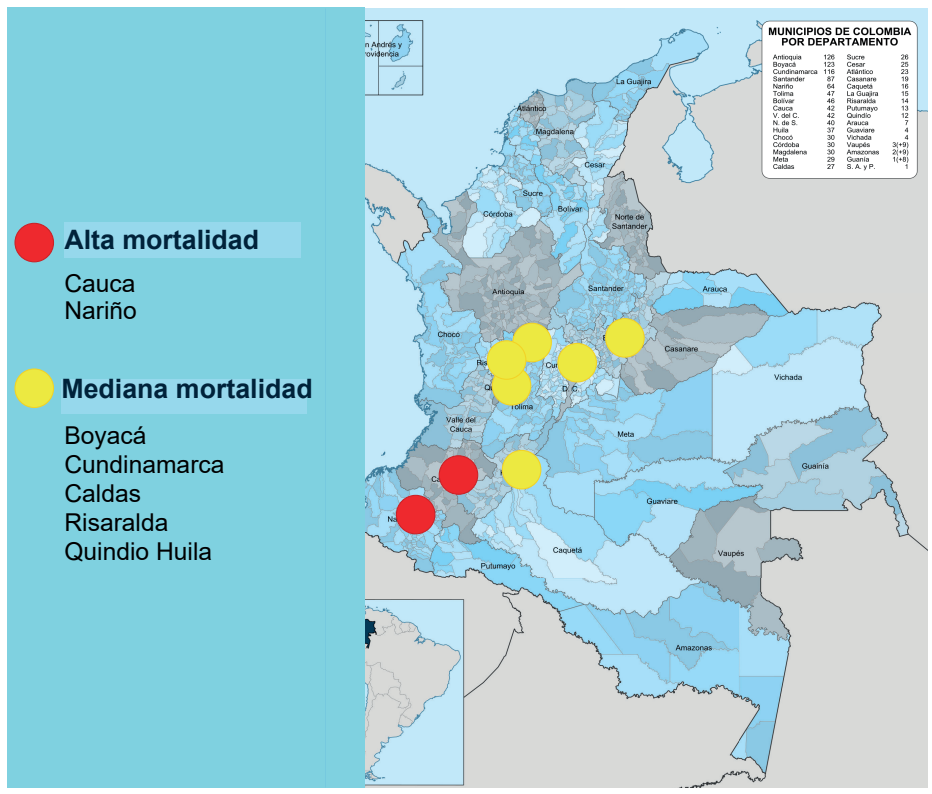

Figura 2. Mortalidad de cáncer gástrico en Colombia
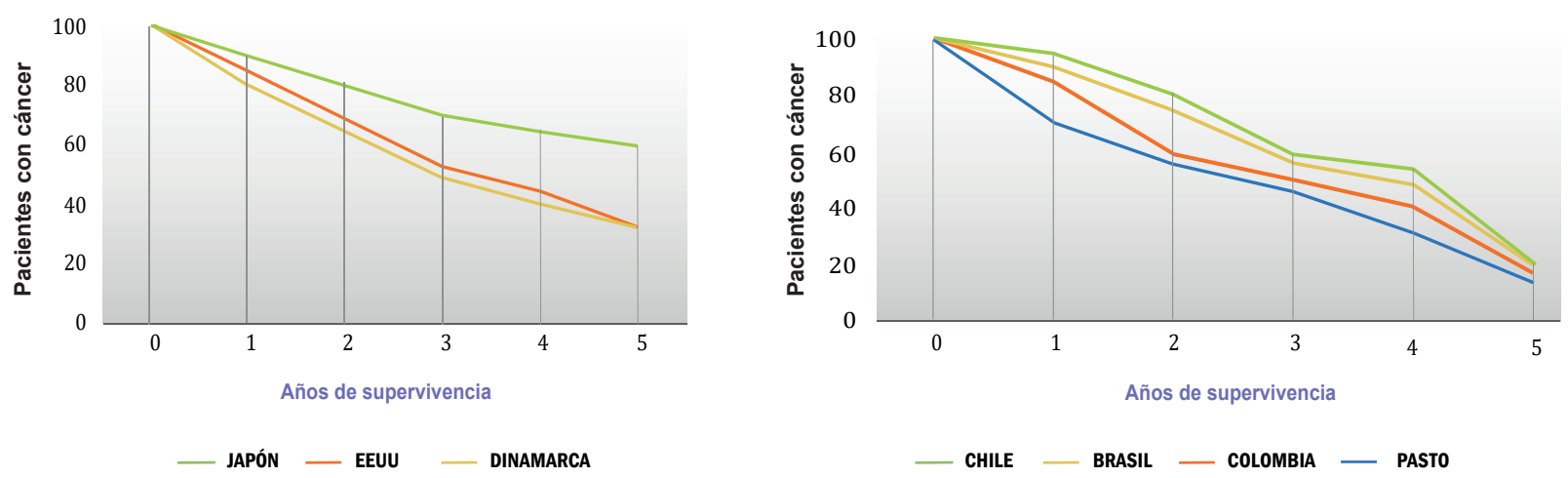

Figura 3. Supervivencia a largo plazo (cinco años)

Fuentes:

Bedoya A. Prevención del cáncer gástrico en Nariño: Una tarea difícil pero factible. Rev. Dirección General de Sanidad Militar 2013: 1; 28-29 Conferencia presentada por el Dr. Bedoya en el VIII Simposio Internacional de cáncer gástrico y Helicobacter pylori. Bello Horizonte, Brasil. Abril, 2018: "URKUNINA 5000 in Colombia"

Los resultados anteriores, nos han llevado a pensar que una de las causas más importantes de la baja supervivencia es la falta de un diagnóstico temprano de cáncer. Existe una gran diferencia entre la intervención que se hace en el Japón para el diagnóstico temprano de cáncer, que alcanza al $73 \%$, con la de nuestro país, donde el diagnóstico temprano es de 2 a $5 \%$ y que influye directamente en la alta tasa de mortalidad por esta causa ${ }^{5,6}$ (figura 4). 


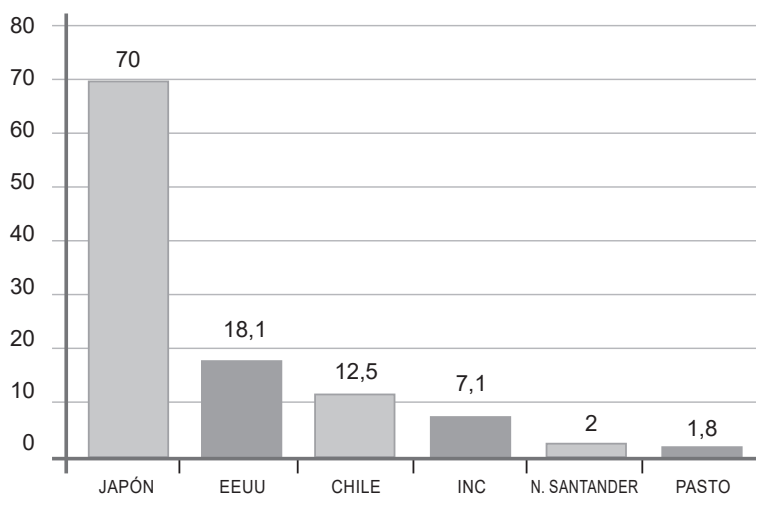

Figura 4. Diagnóstico temprano de cáncer gástrico

Fuentes:

Kenji Y. Hospital Universitario de Chikushi-Fukuoka. Comunicación Personal

Bedoya A. El CIED contra el cáncer gástrico en Nariño. Rev Debates 2009:32;30-1.

Otero W. Cáncer gástrico en Colombia: Un diagnóstico tardío que amerita el compromiso del Estado. Rev Col Gastroenterol. 2008; 23:302-4.

El hecho de vivir por mucho tiempo en la zona andina y poder seguir la historia natural de esta enfermedad por más de 20 años, nos ha permitido conocer mucho mejor la carcinogénesis de esta enfermedad que, indiscutiblemente, es producto de varios factores.

En nuestra región, hemos encontrado cinco factores de riesgo que consideramos principales:

I) alta prevalencia de infección por $H$. pylori, de $60 \%$ en niños y de $90 \%$ en adultos ${ }^{7}$;

2) alta prevalencia $(38,6 \%)$ de lesiones precursoras de malignidad ${ }^{3}$;

3) alto consumo de sal;

4) baja ingestión de micronutrientes y antioxidantes, y

5) la propensión genética.

Tal como lo expuso el autor en la Conferencia del $18^{\circ}$ Congreso de Ciencias Básicas y Especialidades Clínicas del Congreso Intermedio de la ACC, en Bogotá en el mes de abril de 2018 (Órgano para la difusión del programa de formación continuada del médico. Facultad de Medicina. En: AEXMUN. Vol. 23 No I. Abril de 20I8, pg II), estos factores están implicados en $90 \%$ de los casos de cáncer gástrico. De este $90 \%$, el 70\% lo aporta la asociación de lesiones precursoras de malignidad y la infección por $H$. pylori, que se convierten en un dúo mortal en la aparición del cáncer gástrico ${ }^{7}$.

\section{Materiales y métodos}

Aprovechando la Ley de Regalías de Ciencia, Tecnología e Innovación, y en compañía de otras instituciones, se presentó ante Colciencias el proyecto Urkunina 5000, cuyo objetivo general es evaluar la prevalencia de lesiones precursoras de malignidad gástrica y el efecto de la erradicación de $H$. pylori en una población voluntaria de los 55 municipios del área andina del departamento de Nariño. El proyecto contempla cuatro objetivos específicos, de los cuales, el de mayor relevancia es la creación de la primera reserva de material biológico de la población en alto riesgo para cáncer gástrico y de lesiones precursoras de malignidad en el departamento de Nariño, para futuras investigaciones nacionales e internacionales.

Los participantes deben cumplir con los siguientes criterios de inclusión: ser hombres o mujeres entre los 30 y los 70 años de edad, residentes del área urbana o rural de los $55 \mathrm{mu}$ nicipios del Área Andina del departamento de Nariño durante un periodo mínimo de io años, no haberse practicado un estudio endoscópico o histopatológico en el último año, y desear participar en el estudio firmando el consentimiento informado.

Se excluyen del estudio los sujetos con diagnóstico previo de cáncer gástrico u otro tipo de cáncer, pacientes que estén recibiendo tratamiento para enfermedades gastroduodenales, antibióticos con compuestos de bismuto, inhibidores de la bomba de protones, antiinflamatorios no esteroideos (AINE), anticoagulantes o medicamentos para la infección por $H$. pylori en el último mes antes de formar parte del estudio; también, se excluyen personas con antecedentes de cirugía gástrica, o con historia de úlcera gástrica, úlceras sangrantes, várices esofágicas 
u otras condiciones que dificulten el examen de endoscopia.

Según el protocolo para la toma de biopsias, se requieren Io muestras: 5 de antro, 2 de incisura y 3 de cuerpo. Las muestras se distribuyen así:

- Dos biopsias de antro (a $3 \mathrm{~cm}$ del píloro), una de incisura y dos de cuerpo (a $8 \mathrm{~cm}$ del cardias), se destinan para estudio de histología convencional con coloración de hematoxilina y eosina, coloración modificada de Giemsa para detectar H. pylori, y coloración histoquímica con azul alciano para confirmar y clasificar la metaplasia intestinal.

- Una biopsia de antro, una de cisura y otra de cuerpo, se destinan para la extracción de ácidos nucleicos, sin reactivos ni aditamentos, a menos $80^{\circ} \mathrm{C}$, mediante la técnica de laboratorio denominada "NUC seco".

- Una biopsia de antro en guayacolato de glicerol para cultivo y tipificación de la bacteria.

- Una biopsia de antro para validar la prueba de ureasa rápida.

El informe de histopatología ha de reportarse en una escala visual análoga de la versión actualizada del sistema Sidney, que nos permitirá la estadificación en el sistema OLGA/OLGUIN (Operative Link for Gastritis Assessment) (figura 5).

La parte logística de los procedimientos será desarrollada y coordinada por los investigadores del CIEDYN; la interventoría y las actividades administrativas del proyecto serán ejecutadas durante su realización.

La primera fase es la divulgación con los alcaldes, gerentes de empresas sociales del estado (ESE) y médicos de los 55 municipios del área andina, con participación de la gobernación de Nariño y del CIEDYN, y con divulgación y publicidad dirigidas a la población objetivo.

En la segunda fase, se ejecutará el trabajo de campo con la práctica de la endoscopia de vías digestivas altas para recolectar material biológico, y la toma de la muestra de sangre y su separación en los diferentes componentes sanguíneos; se recolectarán muestras de orina, saliva y materia fecal para estudios posteriores. Los componen- tes sanguíneos y las biopsias en "NUC seco", se colocarán en un recipiente a $-80^{\circ} \mathrm{C}$ y se remitirá al biobanco de tumores del Instituto Nacional de Cancerología en Bogotá, donde se mantendrán las muestras a $-80^{\circ} \mathrm{C}$.

Las biopsias para estudio histológico serán remitidas al Departamento de Patología del Hospital San José de Bogotá, donde se mantendrá disponible el informe virtual del diagnóstico, para el control de calidad, elaborado por Blanca Piazuelo de Vanderbilt University.

Los resultados permitirán dividir el grupo de voluntarios en tres subgrupos. El primero, de personas sin lesiones precursoras de malignidad, las cuales serán remitidas a un primer nivel de atención con recomendaciones, entre ellas, el tratamiento de $H$. pylori para quienes lo requieran. El segundo grupo incluye personas con diagnóstico de cáncer gástrico, displasia de alto grado u otras lesiones gástricas, que se remitirán a un tercer nivel de atención. El tercer grupo es el de personas con diagnóstico confirmado de lesiones precursoras de malignidad (gastritis crónica atrófica, metaplasia intestinal o displasia de bajo grado), $\sin H$. pylori, a quienes se les brindará educación nutricional y control endoscópico con toma de biopsia, dos años después del primer resultado.

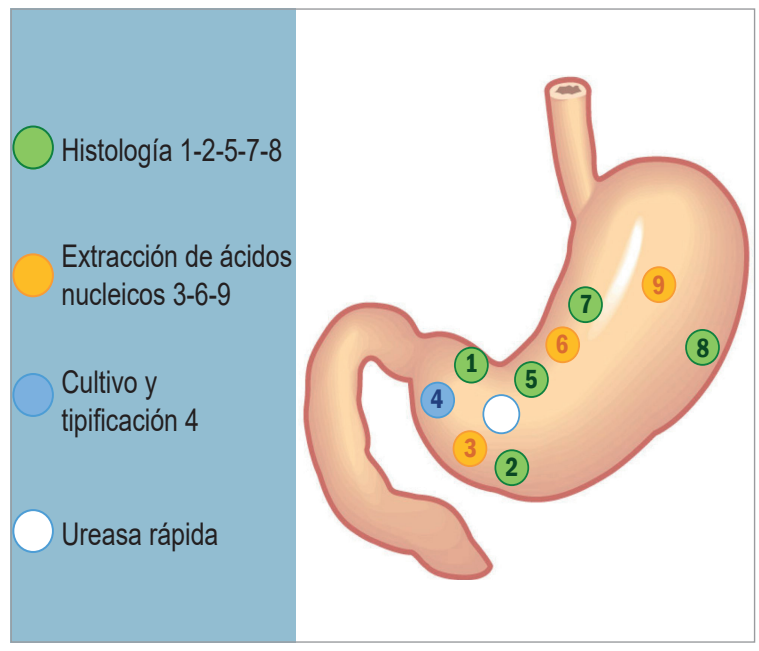

Figura 5. Protocolo de biopsias 
El grupo con $H$. pylori positivo se someterá a tratamiento triconjugado secuencial, con $40 \mathrm{mg}$ diarios de esomeprazol, repartidos en dos dosis durante Io días, $2 \mathrm{~g}$ de amoxicilina en dos dosis durante cinco días, y un gramo de claritromizina en dos dosis durante cinco días. Hay que tener en cuenta que este tratamiento triconjugado secuencial fue el que mejor resultado produjo en la erradicación de H. pylori en las pruebas piloto hechas previamente en nuestra región.

Doce semanas después, se hará la prueba del aliento (Kibion ${ }^{\circledast}$ Dynamic Infra Red Isotope Analyzer) con tecnología de luz infrarroja, cuyos resultados se clasificarán como positivos (presencia de H. pylori) o negativos (ausencia de H. pylori). Un resultado positivo después del tratamiento significa su fracaso; el grupo de investigación hará un análisis minucioso para saber cuáles fueron las causas del fracaso y los pacientes se remitirán para tratamiento de rescate a la respectiva entidad promotora de salud (EPS), con recomendaciones sobre el tratamiento que deben recibir. Estos voluntarios se someterán a control endoscópico e histológico, para analizar el comportamiento de la mucosa gástrica con respecto al primer examen (figura 6).

\section{Impacto}

Las metas al finalizar el estudio, son las siguientes.

I. Caracterización de la población: después de Japón, Nariño sería la única población en el mundo que tiene estudiada histológicamente su población y determinada la prevalencia de lesiones precursoras de malignidad y $H$. pylori, que permitirán orientar acciones pertinentes en promoción y prevención en los municipios de mayor riesgo.

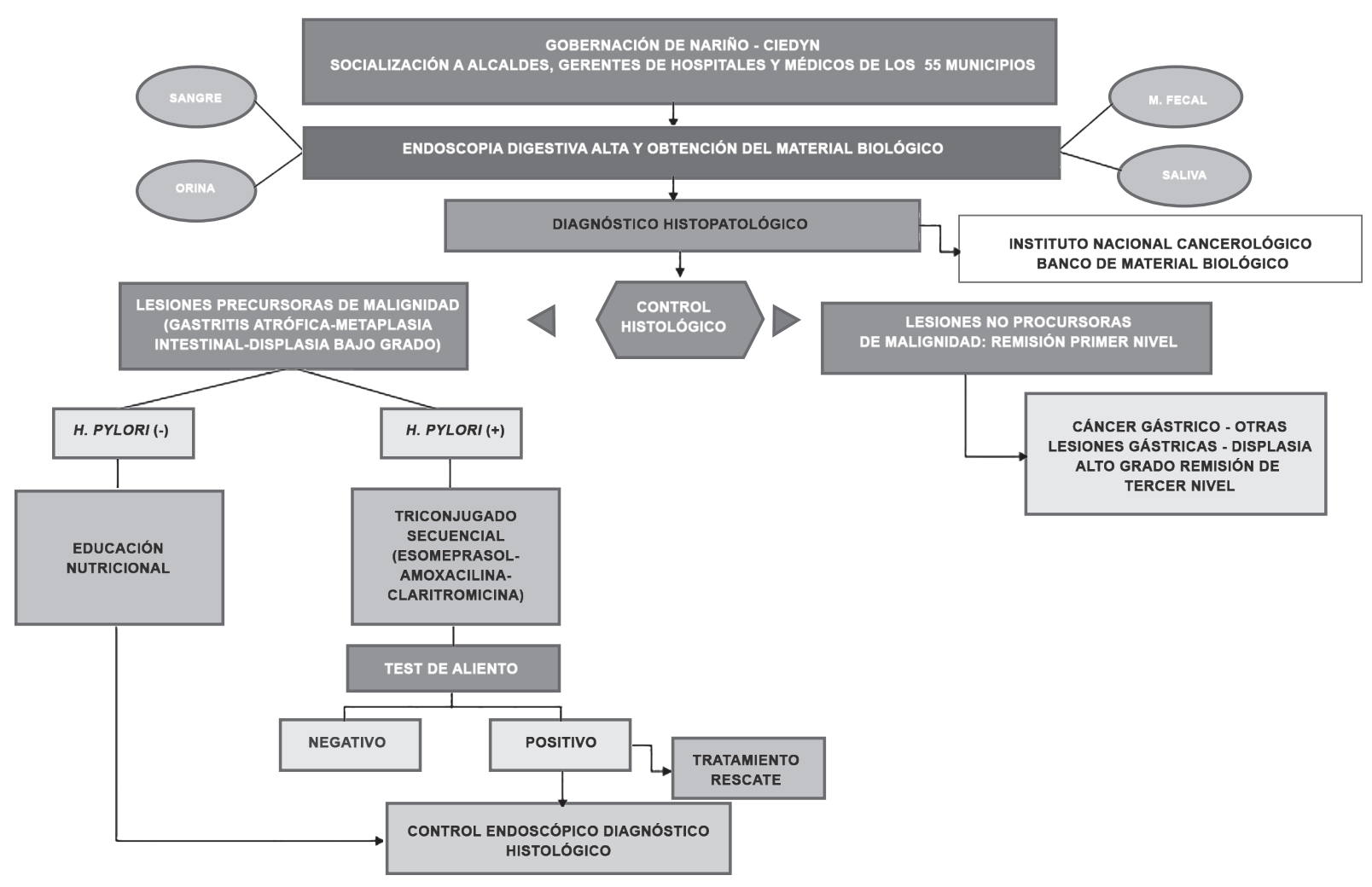

Figura 6. Flujograma de intervención 
2. Medir la efectividad del tratamiento de la erradicación de $H$. pylori, por medio de la prueba del aliento, con el fin de reorientarlo, en caso de que se requiera, o fortalecerlo, si este es efectivo.

3. Tipificación de las cepas bacterianas por municipio, e identificación de posibles mutaciones para analizar la sensibilidad al tratamiento convencional.

4. Creación del biobanco para futuras investigaciones nacionales e internacionales. Se dispondrá de 45.000 muestras de tejido gástrico, 20.000 muestras sanguíneas, más de 3.00o genomas de cepas de H. pylori, y muestras de orina, saliva y materia fecal, lo que nos constituirá en la única región del mundo con una reserva genética de tal magnitud.

5. Elaboración de biomarcadores de bajo costo (para detectar lesiones precursoras de malignidad) y fácil tecnología, proyectada para una segunda fase.

6. Este proyecto contribuirá al fortalecimiento de las políticas públicas para la detección temprana del cáncer gástrico y lesiones precursoras de malignidad.

7. Finalmente, con los resultados de la investigación, se contribuirá al fortalecimiento del conocimiento de la comunidad médica en cuanto a diagnóstico, riesgo, seguimiento, gravedad y tratamiento de las lesiones precursoras de malignidad.

Agradecimiento a los asesores

Pelayo Correa, profesor de Medicina, cátedra de investigación en cáncer Anne Potter Wilson, División de Gastroenterología, Universidad de Vanderbilt, Vanderbilt, Nashville, TN, EE.UU.

María Constanza Camargo, Ph.D., M.Sc., M.H.A., Infections and Inmunoepidemiology Branch, Division of Cancer Epidemiology and Genetics, National Cancer Institute, USA

Guillermo Peres Peres, Associate Profesor of Medicine and Microbiology, New York University
School of Medicine, Departament of Medicine, , New York, EE. UU.

Alba Lucía Trespalacios, Ph.D. M.Sc., profesora titular, directora de línea de investigación, Pontificia Universidad Javeriana, Bogotá, D.C., Colombia

Germán Campusano, profesor de Hematología y Patología; profesor asociado, Universidad de Antioquia, Medellín, Colombia

Arley Gómez López, médico, máster en Biología Molecular y Biotecnología, doctorado en Ciencias Médicas, doctorado en Nanomedicina, Fundación Universitaria Ciencias de la Salud, Bogotá

Anabella Fajardo, bacterióloga, máster en Ciencias Biomédicas, Fundación Ciencias de la Salud, Bogotá, D.C., Colombia

Segundo Morán Villota, investigador titular, Instituto Mexicano de los Seguros Sociales; investigador nacional nivel III, Laboratorio de Investigaciones en Gastro-hepatología, Hospital de Pediatría, Centro Médico Nacional Siglo XXI, México D.F.

Richard M. Peek, Jr., director, División de Gastroenterología; professor of Medicine, Mina Cobb Wallace Chair in Inmunology; professor of Cancer Biology, Vanderlbilt University, Vanderbilt, Nashville, TN, EE.UU.

Keith T. Wilson, professor of Medicine; professor of Pathology, Microbiology and Immunology, Thomas F. First St Chair in Medicine; Professor of Cancer Biology, Vanderbilt University, Vanderbilt, Nashville, TN, EE.UU.

\section{Referencias}

I. Correa P, Piazuelo B. Cáncer gástrico: el enigma colombiano. Rev Col Gastroenterol. 20I0;25:334-7.

2. Correa P. Cáncer gástrico: una enfermedad infecciosa. Rev Col Cirugía. 20II;26:III-7.

3. Bedoya A, Sanzón F, Yépez Y, Santacruz C, Cifuentes C, Calvache D, Bedoya G. A. Prevalencia y severidad de las lesiones precursoras de malignidad en un área de alto riesgo de cáncer gástrico. Pasto, 20I2. Rev Col Gastroenterol. 20I2; 27:275-8I. 
4. Sánchez W, Peña D, García D. Mortalidad por cáncer gástrico en Colombia, informe epidemiológico. Rev Col Cirugía. 2008;23(Sup.):4I.

5. Bedoya A. El CIED contra el cáncer gástrico en Nariño. Rev Debates. 2009;32:30-I.
6. Otero W. Cáncer gástrico en Colombia: Un diagnóstico tardío que amerita el compromiso del Estado. Rev Col Gastroenterol. 2008; 23:302-4.

7. Bedoya A. Prevención del cáncer gástrico en Nariño. Una tarea difícil pero factible. Revista de la Dirección General de Sanidad Militar. 2012; I:28-9. 